\title{
II Programma GICR 2006-2008: continuità ed innovazione
}

\author{
Raffaele Griffo
}

Monaldi Arch Chest Dis 2006; 66: 235-237.

All'indomani del mio insediamento come Presidente della società scientifica Gruppo Italiano di Cardiologia Riabilitativa e Preventiva (GICR), a nome di tutto il Consiglio Direttivo propongo ai Soci alcune riflessioni sui temi che costituiranno il fronte del nostro impegno nel biennio 2006-2008.

Voglio preliminarmente sottolineare, insieme a tutti i componenti del CD, Carmine Riccio vice-presidente, Antonio Castello, Roberto Carlon, Maurizio Ferratini e Carlo Schweiger, che, grazie al lavoro di chi ci ha preceduto, il punto di partenza è molto buono, come testimoniato anche dal grande successo (e l'appassionata partecipazione e dibattito) del nostro congresso di Palermo. Proprio lo svolgimento dei lavori scientifici, dell'Assemblea e delle riunioni di Area ha fornito un ulteriore contributo di idee e riflessioni che questo documento programmatico propone.

Va anche premesso che non sarà facile essere degno successore di Salvatore Pirelli (che comunque nelle vesti di Past-President continuerà a supportarci), non solo per le sue grandi qualità umane, culturali e organizzative che non a caso lo porteranno tra due anni alla presidenza dell'ANMCO, ma sopratutto perché durante il suo mandato ha portato avanti con grande convinzione e con la sua abituale determinazione un chiaro e ben definito progetto: portare la Cardiologia Riabilitativa ad un serrato confronto culturale e organizzativo con l'intera filiera assistenziale del paziente cardiopatico, in particolare con la Cardiologia dell'acuzia, cercando di rafforzare il ruolo di interlocutore scientificamente e professionalmente credibile della nostra Società, da tenere quindi nel giusto conto nella costruzione dei percorsi assistenziali del cardiopatico completi, efficaci e condivisi.

Per completare questo cammino di crescita culturale ed organizzativa, bisogna ora procedere su due fronti, uno interno al GICR e uno esterno, nel rapporto con le altre società scientifiche e con le Istituzioni.

\section{La politica interna}

Sono profondamente convinto che la credibilità di una Società Scientifica, e quindi anche della nostra, si fondi principalmente sul fatto che essa sia realmente rappresentativa di un consistente numero di strutture e professionisti che operano nel settore ad ogni livello di complessità sia assistenziale che organizzativa.

Questo requisito è oggi solo in parte soddisfatto: molte strutture, in particolare le più semplici e diffu- se, non sono adeguatamente rappresentate, molti cardiologi che pur lavorano quotidianamente nel settore non risultano iscritti al Gruppo, molti tra gli iscritti o non versano regolarmente la modesta quota associativa o comunque non sentono la vita associativa al centro dell'attenzione, la presenza di altri professionisti del team preventivo e riabilitativo, infermieri, fisioterapisti, dietisti, rimane, ad eccezione degli psicologi, assolutamente marginale e senza organizzazione e rappresentatività. Quali sono i motivi di questa insoddisfacente situazione?

Una delle possibili risposte è legata all'analisi delle aspettative di chiunque valuti se iscriversi o meno ad una Società Scientifica, e cioè l'offerta di quest'ultima in termini di servizi, aggiornamento e crescita professionale e di coinvolgimento nella vita associativa e culturale. Credo che sia necessario impegnarsi a fondo perché queste aspettative non vadano deluse. Provo a fare un elenco sommario di ciò che potrebbe essere fatto a questo scopo:

1) Potenziare il sito Web, ormai protagonista indiscusso della circolazione in tempo reale delle informazioni e delle opinioni. Il nostro sito deve diventare per gli associati (e non solo) un interessante ed efficace strumento di consultazione, di aggiornamento, di confronto e circolazione di idee connesse alla realtà della cardiologia riabilitativa. Per questo deve dotarsi non solo di una grafica più attrattiva e al passo con la rapida evoluzione del mezzo, ma anche di strumenti di aggiornamento e di interazione più immediati ed efficaci. Nella ipotesi di realizzare un reale strumento di aggiornamento e di reperimento di informazione e materiale e in prospettiva futura di formazione a distanza, ritengo che il sito debba anche proporsi come mezzo per l'aggiornamento on-line dei dati dei Centri, rendendo così sempre completa e aggiornata la realtà operativa della cardiologia riabilitativa in Italia, e come sede per forum per commenti su documenti e Linee Guida (comprese, per prime, le LLGG sulla prevenzione e riabilitazione cardiologica). Avendo ben presenti questi obbiettivi, sarà chiesto a tutti $e$, in modo più formale e vincolante ai delegati regionali, ai rappresentanti di area non medica, ai futuri gruppi di lavoro e certamente al $\mathrm{CD}$ (con la proposizione di una newsletter periodica) di collaborare in modo costante alla sua vita, per renderlo così la vera "house horgan" della società. Andranno anche ricercate a questo scopo forme stabili di finanziamento e definiti accordi precisi con l'Agenzia incaricata della gestione operativa del sito. 
2) Creare occasioni formative agili, interattive, pratiche e fruibili su tutto il territorio nazionale. A questo scopo andranno potenziate le riunioni regionali o macro-regionali sulla scorta di quanto abbiamo iniziato a fare, con modalità itinerante e grande successo, nel biennio scorso:

a) con Area Prevenzione ANMCO con il "Corso clinico pratico di Cardiologia Preventiva e Riabilitativa", basato esclusivamente su casi clinici interattivi e che ha già visto quest'anno tre edizioni (a Bologna, Cremona e Napoli), e che, sempre grazie ad un grant incondizionato di Guidotti, ne vedrà altrettante nel 2007; e

b) con il progetto "COREX: corso sulla stratificazione prognostica nel post-IMA e strategie di prevenzione secondaria", centrato sull'obbiettivo di disseminare le Linee Guida GICR-ISS-ASSR, di discutere gli ostacoli e le barriere "locali" alla prevenzione e riabilitazione, e di formare i cardiologi nelle tecniche di comunicazione medico-paziente (con la tutorship dei nostri psicologi), già tenutosi a Napoli, Genova, Brescia, Bologna, Roma e Catania, e che, sempre grazie al supporto incondizionato di SPA, verrà reiterato nel 2007 in altre sei sedi.

A queste iniziative saranno affiancati i consueti convegni regionali, grazie all'impegno e all'iniziativa dei referenti e dei nostri soci, che saranno un ulteriore occasione per una conoscenza diretta delle problematiche locali e di confronto con le altre società scientifiche.

3) Agevolare gli stages formativi di tutte le figure professionali del team riabilitativo presso strutture accreditate. La richiesta e il bisogno da parte di tutte le figure professionali per una formazione e un aggiornamento "sul campo" è emersa con grande evidenza nelle Assemblee di Palermo. Bisognerà quindi prevedere l'individuazione, con criteri di trasparenza, di una rete di centri qualificati e, in casi selezionati, di forme di supporto economico per facilitarne la fruibilità, specie ai professionisti non medici.

4) Dare maggiore spazio propositivo e consultivo ai Delegati Regionali ma nel contempo chiederne un più puntuale monitoraggio delle attività. Il rapporto centro-periferia e periferia-periferia è da sempre una criticità della nostra associazione: il ruolo e l'impegno del singolo Delegato è decisivo come dimostrato da alcuni brillanti esempi nell'ultimo biennio. Il CD si ritiene già ora impegnato ad intervenire, fornendo una precisa descrizione dei compiti (raccolta di Leggi Regionali concernenti la riabilitazione, inserimento nei Piani Regionali di Prevenzione, analisi della situazione regionale e delle sue criticità, attivazione di programmi culturali e apertura di canali stabili di collegamento con le rappresentanza locali delle altre società scientifiche cardiologiche), assicurando audit periodici, ascolto (con $\mathrm{CD}$ allargati ai delegati regionali in piccoli gruppi per volta), e supporto segretariale.

5) Dare voce e spazio alle Aree professionali. Il congresso di Palermo è stato per le Aree dei Fisioterapisti, degli Infermieri e dei Dietisti una grande (ma purtroppo anche unica) occasione di incontro e dibattito. In questo settore, fatta eccezione per gli Psicologi, c'è molto da fare e la strada da intraprendere è simile a quella prevista per i delegati regionali, con l'individuazione, per il momento avocata al $\mathrm{CD}$, di referenti per le singole aree che aggreghino gli interessati, raccolgano le loro istanze e propongano iniziative, contando sul supporto organizzativo del GICR.

6) Aumentare il coinvolgimento dei soci istituendo numerosi, ma ristretti (e quindi agili), gruppi di lavoro, anche interprofessionali, per mettere a fuoco argomenti sia culturali (ricerca e formazione) che organizzativi, con un mandato chiaro e termini temporali precisi. Attiveremo gruppi di lavoro che propongano, su delega e controllo del Consiglio Direttivo:

a) raccomandazioni-strumenti di lavoro per l'attività fisica (dal recupero della autonomie al training vero e proprio),

b) nuovi modelli organizzativi, comunicazionali e motivazionali di gestione del programma di educazione sanitaria e terapeutica,

c) strumenti di valutazione ed intervento che possano confluire in una Cartella clinica standard di Riabilitazione Cardiologica,

d) percorsi riabilitativi del paziente complesso e con Scompenso Cardiaco,

e) progetti di ricerca clinica concretamente realizzabili sul modello di registri e studi clinici e proposte di temi per il nostro Congresso Nazionale e per altre attività di formazione in collaborazione con altre società (e mi riferisco all'area scompenso ANMCO, alla cardiochirurgia, all'interventistica, all'epidemiologia, ai metabolisti, ai geriatri e, perché no, ai fisiatri),

f) definizione e messa in atto di specifici e continuativi rapporti con le Istituzioni.

Gli elaborati di questi gruppi di lavoro potrebbero poi confluire, per una massima diffusione e condivisione, in un appuntamento annuale da effettuare nell'anno in cui non si svolge il Congresso Nazionale. In questa medesima occasione si potrebbe riservare uno spazio per controversie con le altre "anime" della cardiologia e della medicina. Questo incontro, a mio parere, può rappresentare un formidabile strumento di crescita culturale e associativa.

\section{La politica verso l'esterno}

Lo statuto è stato modificato per consentire al GICR di possedere gli strumenti per essere riconosciuta come Società Scientifica sia a livello ministeriale sia per aderire, con piena dignità e, soprattutto, rappresentatività alla Federazione Italiana di Cardiologia. Ma non basta: non è sufficiente operare solo sul piano formale, è indispensabile lavorare su quello sostanziale: solo una Società forte dal punto di vista partecipativo e rappresentativa di un movimento in crescita culturale e organizzativa può essere garante per la Cardiologia italiana della qualità dei suoi interventi.

Solo in questo modo il GICR, potrà proporsi nei fatti, e non nella teoria, come la "casa comune" della Prevenzione e Riabilitazione Cardiovascolare nel 
nostro Paese, presentandosi ad un confronto costruttivo con tutte le altre componenti in campo con l'obbiettivo di unificarne la rappresentanza o di costituire il nerbo di un nuovo soggetto, seguendo la strada già percorsa dalla Società Europea di Cardiologia con la costituzione dell'EACPR sulle ceneri dei working group. Interlocutori d'obbligo in questo percorso sono:

a) l'Area Prevenzione dell'ANMCO (compresa la sua anima "epidemiologica"), con la quale, grazie al precedente Direttivo, si sono fatti già concreti e significativi passi per la condivisione di progetti formativi e scientifici, in cui da tempo è in atto una forte integrazione a livello di soci e una vera e propria compartecipazione a livello di Direttivi, con la presenza del co-chairman dell'Area e del vice-Presidente GICR ai rispettivi $\mathrm{CD}$, e

b) il corrispondente Gruppo di Studio della SIC che ha già manifestato senza incertezze attraverso il coordinatore della Commissione Gruppi di Studio della SIC, Salvatore Novo, una precisa volontà di confluire con l'Area Prevenzione dell'ANMCO e successivamente nella nostra società.

Questi rapporti "privilegiati” non escludono altri soggetti, a patto che dimostrino di essere portatori di contenuti e rappresentatività concrete.

Sempre su questo versante "politico" proseguiremo, con il supporto della FIC, il confronto a livello ministeriale (e regionale, in quelle realtà in cui fosse necessario) con le rappresentanze dei fisiatri per mettere fine, una volta per tutte, alla stucchevole e strumentale querelle su chi abbia la titolarità della riabilitazione specialistica cardiologica. Pur ribadendo che esiste tra le due specialità un ampio spazio di interazione nell'esclusivo interesse di malati sempre più anziani, disautonomi, fragili e con sempre maggiore complessità assistenziale, nel contempo sarà riaffermato in tutte le sedi che le nostre competenze, esperienze e conoscenze nel settore sono tali da rendere assolutamente irrealistica e pericolosa, per il paziente e per la sanità italiana, la gestione del percorso riabilitativo cardiologico da parte dei fisiatri. La questione appare ancor più irragionevole e fuori dalla realtà sanitaria attuale, caratterizzata dalla centralità della persona malata e dalla multi-professionalità dei team assistenziali, nei quali la titolarità "concreta" è di volta in volta assunta dalla figura maggiormente coinvolta e competente sui bisogni prevalenti del singolo paziente.

Un altro fronte sul quale bisognerà prestare grande attenzione è quello della Primary Care, vero snodo ineludibile per assicurare ai nostri pazienti la continuità assistenziale e percorsi diagnostici e terapeutici condivisi.

La credibilità di una Società Scientifica come la nostra è anche l'essere ben identificata dalle Istituzioni. A questo proposito il GICR deve far valere la propria indiscussa leadership culturale e organizzativa nel settore, già dimostrata con l'ISS e l'ASSR in occasione della stesura delle Linee Guida e con alcuni assessorati regionali nell'elaborazione dei Piani Regionali di Prevenzione sull'alto rischio e sulle recidive. Questo riconoscimento porterà ad essere coinvolti sistematicamente nella elaborazione di Linee Guida, di percorsi diagnostico-assistenziali delle patologie cardiovascolari, di documenti di programmazione sanitaria e di riorganizzazione delle reti ospedaliere ed extra-ospedaliere. Inoltre, per tali competenze, il GICR dovrà essere coinvolto, anche con il supporto della FIC, nel percorso formativo degli specialisti cardiologi come previsto dalla recente revisione dei contenuti del quinquennio di studi, e dei laureandi in scienze infermieristiche e fisioterapistiche.

Aggiungo infine che la visibilità del GICR come interlocutore serio, credibile e rappresentativo deve essere forte anche per l'Industria: è evidente che le nostre iniziative sono possibili solo se sostenibili economicamente, ma è altrettanto chiaro che esse stesse, così come avviene per le altre società scientifiche, diventino uno strumento di grande impatto per assicurare un accesso stabile e non più "sofferto" a risorse per le attività più squisitamente di ricerca e formazione.

Per concludere, il GICR ha compiuto negli ultimi dieci anni straordinari progressi. Fare il bilancio dei grandi successi e della crescita complessiva del Gruppo non è certo mio compito, anche se ne sono stato in buona parte testimone diretto: il grande lavoro fatto da chi mi ha preceduto (voglio citare, oltre al presidente uscente, almeno Pantaleo Giannuzzi, Carmine Chieffo e Francesco Fattirolli), ha ben preparato il terreno. È venuto il momento di fare un'ulteriore passo in avanti.

Il momento socio-sanitario, culturale, "politico" che finalmente pone in grande risalto la riabilitazione e prevenzione e le propizie circostanze "ambientali" (e mi riferisco ai tanti nostri amici ai vertici di Società e Associazioni Cardiologiche, da Salvatore Pirelli presidente designato ANMCO, a Carmine Chieffo vice-presidente dell'HCF, a Massimo Piepoli, vice-coordinatore del Gruppo di Studio SIC su Fisiologia dell'esercizio, Cardiologia dello Sport e Riabilitazione Cardiovascolare e membro del Nucleus della "Cardiac Rehabilitation Section" della EACPR, a tanti altri) sono straordinariamente favorevoli per il nostro settore di attività cardiologica. Sta a tutti noi, Consiglio Direttivo, delegati regionali ma anche, e soprattutto, ad ognuno dei soci, saperlo cogliere.

Buon lavoro a tutti 